\title{
Laplace operators on Sasaki-Einstein manifolds
}

\author{
Johannes Schmude \\ Department of Physics, Universidad de Oviedo, \\ 3300\%, Oviedo, Spain \\ RIKEN Nishina Center, \\ Saitama 351-0198, Japan \\ E-mail: schmudejohannes@uniovi.es
}

Abstract: We decompose the de Rham Laplacian on Sasaki-Einstein manifolds as a sum over mostly positive definite terms. An immediate consequence are lower bounds on its spectrum. These bounds constitute a supergravity equivalent of the unitarity bounds in dual superconformal field theories. The proof uses a generalisation of Kähler identities to the Sasaki-Einstein case.

KEYwords: Differential and Algebraic Geometry, AdS-CFT Correspondence

ARXIV EPRINT: 1308.1027 


\section{Contents}

1 The proof 1

1.1 Exterior calculus on Sasaki-Einstein manifolds 2

1.2 Lefschetz decomposition 4

1.3 Kähler-like identities 5

$\begin{array}{ll}1.4 \text { Beyond Kähler identities? } & 7\end{array}$

$\begin{array}{lll}2 & \text { Motivation and applications } & 7\end{array}$

$\begin{array}{ll}\text { A The identities } & 9\end{array}$

A textbook result in Kähler geometry relates the de Rham with the Dolbeault Laplacian, $\Delta=2 \Delta_{\bar{\partial}}$. The main result of this note is a similar identity for Sasaki-Einstein manifolds:

$$
\Delta=2 \Delta_{\bar{\partial}_{B}}-£_{\xi}^{2}-2 \imath\left(n-d^{0}\right) £_{\xi}+2 L \Lambda+2\left(n-d^{0}\right) L_{\eta} \Lambda_{\eta}+2 \imath\left(L_{\eta} \bar{\partial}_{B}^{*}-\bar{\partial}_{B} \Lambda_{\eta}\right) .
$$

The right hand side of equation (1) features the Lefschetz operator, the action of the Reeb vector, the tangential Cauchy-Riemann operator as well as their adjoints. Full definitions will be given in section 1 . The equation $\Delta=2 \Delta_{\bar{\partial}}$ can be derived from the Kähler identities, commutators between the Dolbeault and Lefschetz operators and their adjoints [1,2]. Our proof of (1) will follow a similar route; we will obtain Kähler-like identities that are valid on Sasaki-Einstein manifolds. These are summarized in appendix A.

This note is written with two audiences in mind: physicists working in gauge/string duality or supergravity and mathematicians interested in Sasaki-Einstein geometry. Therefore we split the discussion into two separate parts, giving a proof of both (1) and the identities in section 1, while discussing their motivation by and relevance to physics in section 2 . Readers who want to focus on the mathematical aspects can ignore section 2 . Those not interested in the full proof should read section 1 up to equations (1.4) before skipping ahead to section 2 .

\section{The proof}

The tangential Cauchy-Riemann operator $\bar{\partial}_{B}$ and the associated Kohn-Rossi cohomology groups $H_{\bar{\partial}_{B}}^{p, q}(S)$ were first introduced in [3-5]. Given a complex manifold with boundary, Lewy, Kohn, and Rossi considered under what circumstances functions on the boundary can be extended to holomorphic functions in the bulk. They have to satisfy the projection of the Cauchy-Riemann equations onto the boundary, hence the name for $\bar{\partial}_{B}$. The KohnRossi cohomology groups feature also in the work by Yau and collaborators on the complex plateau problem [6-8]. This concerns the question when a real manifold is also the boundary of a complex manifold. 
The tangential Cauchy-Riemann operator has properties akin to those of a Dolbeault operator on a Kähler manifold. Yet it is not the only differential operator on a SasakiEinstein manifold with this characteristic. The simplest example is the basic differential $\bar{\partial}_{\text {bsc }}$, which arises in the context of basic forms. ${ }^{1}$ These are transverse to the Reeb vector and carry no charge under the Lie derivative along the Reeb. If one drops the zero-charge condition, it is possible to introduce a transverse differential $\bar{\partial}_{T}$. This has been studied by Tievsky [9], who obtained transverse Kähler identities, which are a special case of the identities derived in section 1 and summarized in section A. Note that we will refer to transverse forms as horizontal.

In what follows, we will give a full proof of (1) after setting the stage by giving all necessary definitions. Since the proof is based on the equivalent considerations in the Kähler case, our discussion will follow $[1,2]$ very closely.

\subsection{Exterior calculus on Sasaki-Einstein manifolds}

Consider a $d=2 n+1$ dimensional Sasaki-Einstein manifold $S$. Given the Reeb vector $\xi$ and the contact form $\eta$, the tangent bundle splits as $T S=D \oplus L_{\xi} \cdot{ }^{2}$ Furthermore, there is a two-form $J=\frac{1}{2} d \eta$ with $i_{\xi} J=0 . J$ defines an endomorphism on $T S$ which satisfies $J^{2}=-1+\xi \otimes \eta$. Since $\eta(D)=0$, one can decompose the complexified tangent bundle as $T_{\mathbb{C}} S=(\mathbb{C} \otimes D)^{1,0} \oplus(\mathbb{C} \otimes D)^{0,1} \oplus(\mathbb{C} \otimes \xi)$. This in turn induces a corresponding decomposition on the complexified cotangent bundle

$$
T_{\mathbb{C}}^{*} S=\Omega^{1,0} \oplus \Omega^{0,1} \oplus(\mathbb{C} \otimes \eta)
$$

which also extends to the exterior algebra

$$
\Omega_{\mathbb{C}}^{*}=\bigoplus_{p, q} \Omega^{p, q} \wedge(1 \oplus \eta)
$$

Elements of $\Omega_{\mathbb{C}}^{*}$ that vanish under the action of $i_{\xi}$ are called horizontal, while those annihilated by $\eta \wedge$ are vertical.

The decomposition (1.1) induces a decomposition of the exterior derivative,

$$
d=\partial_{B}+\bar{\partial}_{B}+£_{\xi} \eta \wedge
$$

$\bar{\partial}_{B}$ is the tangential Cauchy-Riemann operator. $\partial_{B}$ and $\bar{\partial}_{B}$ satisfy $\left\{\partial_{B}, \bar{\partial}_{B}\right\}=-2 J \wedge £_{\xi}$ as well as $\partial_{B}^{2}=\bar{\partial}_{B}^{2}=0$. The sequence

$$
\ldots \stackrel{\bar{\partial}_{B}}{\longrightarrow} \Omega^{p, q-1} \stackrel{\bar{\partial}_{B}}{\longrightarrow} \Omega^{p, q} \stackrel{\bar{\partial}_{B}}{\longrightarrow} \Omega^{p, q+1} \stackrel{\bar{\partial}_{B}}{\longrightarrow} \ldots
$$

gives rise to the Kohn-Rossi cohomology groups $H_{\bar{\partial}_{B}}^{p, q}(S)$. Continuing with the theme of generalizing concepts from Kähler geometry to Sasaki-Einstein manifolds, we define the

\footnotetext{
${ }^{1}$ Our notation is non-standard. The basic differential is often denoted as $\bar{\partial}_{B}$, with the tangential CauchyRiemann operator being $\bar{\partial}_{b}$.

${ }^{2} L_{\xi}$ is the line tangent to $\xi$. In what follows we will set $L_{\xi}=\xi$ and $L_{\xi}^{*}=\eta$. See section 1 of [10] for a review of Sasaki-Einstein geometry.
} 
Lefschetz operator $L: \Omega_{\mathbb{C}}^{k} \rightarrow \Omega_{\mathbb{C}}^{k+2}$ via $\alpha \mapsto J \wedge \alpha$ and the Reeb operator $L_{\eta}: \Omega_{\mathbb{C}}^{k} \rightarrow \Omega_{C}^{k+1}$ as $\alpha \mapsto \eta \wedge \alpha$.

Introducing the Hodge star ${ }^{3}$

$$
\star \bar{\alpha} \wedge \beta=\frac{1}{p !} \bar{\alpha}^{m_{1} \ldots m_{p}} \beta_{m_{1} \ldots m_{p}} \operatorname{vol}=\langle\alpha, \beta\rangle \text { vol },
$$

allows us to define adjoints for the above operators when acting on $\Omega_{\mathbb{C}}^{k}$ :

$$
\begin{aligned}
d^{*} & =(-1)^{k} \star d \star, \\
\partial_{B}^{*} & =(-1)^{k} \star \bar{\partial}_{B} \star, \\
\bar{\partial}_{B}^{*} & =(-1)^{k} \star \partial_{B} \star, \\
\Lambda & \left.=L^{*}=\star L \star=J\right\lrcorner, \\
\Lambda_{\eta} & =L_{\eta}^{*}=(-1)^{k+1} \star L_{\eta} \star=i_{\xi}, \\
\left(L_{\eta} £_{\xi}\right)^{*} & =-\Lambda_{\eta} £_{\xi} .
\end{aligned}
$$

Recall that on odd-dimensional manifolds $\star$ satisfies $\star \star=1$.

When restricted to $D$, the action of $J$ becomes that of an almost complex structure $\mathcal{I}$ which acts as $\mathcal{I}(\alpha)=J_{m}^{n} \alpha_{n} d x^{m}$ and $\mathcal{I}(X)=X^{m} J_{m}{ }^{n} \partial_{n}$. Of course $\Omega^{1,0}=\{\alpha \in$ $\left.\Omega^{1} \mid \mathcal{I}(\omega)=\imath \omega\right\}$. We also define

$$
\boldsymbol{I}=\sum_{p, q} \imath^{p-q} \Pi^{p, q}
$$

which makes use of the projection $\Pi^{p, q}: \Omega_{\mathbb{C}}^{*} \rightarrow \Omega^{p, q}$.

It will turn out useful to distinguish between the rank of a form on $\Omega_{\mathbb{C}}^{*}$ and on $\bigwedge^{*} D^{*}$. Hence we define the operator $d^{0}$ on $\Omega_{\mathbb{C}}^{*}$ via

$$
\left.d^{0}\right|_{\wedge^{k} D^{*} \wedge(1 \oplus \eta)}=k \cdot \mathrm{id} .
$$

By definition, $d^{0}$ commutes with $L_{\eta}$. A first example of the uses of $d^{0}$ is given by the notion of primitive forms. $\alpha \in \Omega_{\mathbb{C}}^{*}$ with $d^{0} \leq n$ is primitive if and only if $\Lambda \alpha=0$. Essentially, the idea of primitivity on $\Lambda^{*} D^{*}$ is the same as on Kähler manifolds, the contact one-form just comes along for the ride and there is in no difference between horizontal and vertical forms. We define $P^{k}$ as the set of primitive elements of $\bigwedge^{k} D^{*}$.

Next we introduce an orthonormal frame $e^{i}$ on $D^{*}$. Defining $z^{i}=e^{2 i-1}+\imath e^{2 i}$ and imposing $\mathcal{I}\left(z^{i}\right)=\imath z^{i}$, consistency requires that $\mathcal{I}\left(e^{2 i-1}\right)=-e^{2 i}$ and $\mathcal{I}\left(e^{2 i}\right)=e^{2 i-1}$. Then

$$
J=\sum_{i}^{n} e^{2 i-1} \wedge e^{2 i}=\frac{\imath}{2} \sum_{i}^{n} z^{i} \wedge \bar{z}^{i} .
$$

Defining $e^{2 n+1}=\eta$, one finds vol $=\operatorname{vol}_{D^{*}} \wedge e^{2 n+1}=\frac{1}{n !} J^{n} \wedge \eta$.

In what follows, we will make use of two results concerning the Hodge star. To begin, assume that $(V,\langle\rangle$,$) is a Euclidean vector space admiting a decomposition V=W_{1} \oplus W_{2}$

\footnotetext{
${ }^{3}$ In components

$$
\star \alpha_{m_{1} \ldots m_{p}}=\frac{\sqrt{g}}{p !} \epsilon_{m_{1} \ldots m_{d-p}}^{n_{1} \ldots n_{p}} \alpha_{n_{1} \ldots n_{p}} .
$$
}


that is compatible with the metric $\langle$,$\rangle . For simplicity we assume that \operatorname{dim}_{\mathbb{R}} W_{i} \in 2 \mathbb{Z}$. The metrics $\langle,\rangle_{i}$ induce Hodge star operators $\bullet$ i, $i=1,2$. Then $\bigwedge^{*} V^{*}=\bigwedge W_{1}^{*} \otimes \bigwedge W_{2}^{*}$, and for $\alpha_{i} \in \bigwedge^{k_{i}} W_{i}^{*}$, the Hodge dual on $\bigwedge^{*} V^{*}, \bullet$, threads as

$$
\text { • }\left(\alpha_{1} \otimes \alpha_{2}\right)=(-1)^{k_{1} k_{2}} \bullet{ }_{1} \alpha_{1} \otimes \bullet_{2} \alpha_{2},
$$

since $\left(\beta_{i} \in W_{i}\right)$

$$
\begin{aligned}
\bullet\left(\alpha_{1} \otimes \alpha_{2}\right) \wedge\left(\beta_{1} \otimes \beta_{2}\right) & =\left\langle\alpha_{1}, \beta_{1}\right\rangle_{1}\left\langle\alpha_{2}, \beta_{2}\right\rangle_{2} \operatorname{vol}_{1} \operatorname{vol}_{2} \\
& =(-1)^{k_{1} k_{2}} \bullet_{1} \alpha_{1} \wedge \bullet_{2} \alpha_{2} \wedge \beta_{1} \wedge \beta_{2} .
\end{aligned}
$$

One can use identical considerations to decompose the action of $\star$ on $\Omega_{\mathbb{C}}^{*}$ into seperate operations on $D^{*}$ and $\eta$. Introducing a hodge dual $\bullet$ on $D^{*}$, one finds

$$
\left.\star\right|_{\wedge^{*} D^{*}}=L_{\eta} \bullet,\left.\quad \star\right|_{\wedge^{*} D^{*} \wedge \eta}=\bullet(-1)^{d^{0}} \Lambda_{\eta} .
$$

\subsection{Lefschetz decomposition}

The starting point for our discussion of Lefschetz decomposition is the commutator

$$
[L, \Lambda]=\left(d^{0}-n\right)
$$

The proof is via induction in $n$. Consider $d=3, n=1$. Then $\Omega_{\mathbb{C}}^{*}$ is spanned by $\left\{1, \eta, \mu_{i}, J, J \wedge \eta\right\}$ where $\mu_{i} \in \Omega^{1,0} \oplus \Omega^{0,1}$ and both $\mu_{i}$ are annihilated by $L$ and $\Lambda$. Then $\Lambda J=1$ and thus $\left.[L, \Lambda]\right|_{\Omega_{\mathbb{C}}^{0}}=-1,\left.[L, \Lambda]\right|_{\eta}=-1,\left.[L, \Lambda]\right|_{D^{*}=0},\left.[L, \Lambda]\right|_{\Omega^{1,1}}=1$, and $\left.[L, \Lambda]\right|_{\Omega_{\mathbb{C}}^{3}}=1$. Hence

$$
\left.[L, \Lambda]\right|_{\wedge^{k} D^{*} \wedge(1 \oplus \eta)}=(k-1), \quad k=0,1,2,
$$

as claimed. The induction then proceeds as in [1].

Eq. (1.7) generalizes to

$$
\left.\left[L^{i}, \Lambda\right]\right|_{\Lambda^{k} D^{*} \wedge(1 \oplus \eta)}=\imath(k-n+i-1) L^{i-1} .
$$

Again the proof is a copy of that in [1].

To proceed we follow [2]. Restricting to $\bigwedge^{*} D^{*}$ one can copy all results from proposition 6.20 to lemma 6.24. The most important of these results is Lefschetz decomposition. Given $\alpha \in \bigwedge^{k} D^{*}$, there is a unique decomposition

$$
\alpha=\sum_{r} L^{r} \alpha_{r}, \quad \alpha \in P^{k-2 r} .
$$

The decomposition is compatible with the bidigree decomposition and with the decomposition into horizontal and vertical components. Moreover,

$$
L^{n-k}: \bigwedge^{k} D^{*} \rightarrow \bigwedge^{2 n-k} D^{*}
$$

is an isomorphism and the primitivity condition is equivalent to $L^{n-k+1} \alpha=0$. 
The Lefschetz decomposition becomes incredibly useful when used together with the Bidigree decomposition, equation (1.6) and the identity

$$
\begin{aligned}
\forall \alpha \in P^{k}, \quad \bullet L^{j} \alpha & =F(n, j, k) L^{n-k-j} \boldsymbol{I}(\alpha), \\
F(n, j, k) & =(-1)^{\frac{k(k-1)}{2}} \frac{j !}{(n-k-j) !} .
\end{aligned}
$$

Since no differential operators are involved and $\alpha \in \bigwedge^{k} D^{*}$, one can copy the proof in [1] after adjusting for conventions. Once the dust settles, the only difference is in the $k$ dependent prefactor.

\subsection{Kähler-like identities}

We are finally in a position to make use of the previous results and calculate the (anti-) commutators. The results are in summarized in table 1. A number of identities are fairly obvious:

$$
\begin{aligned}
0 & =\left[\partial_{B}, L\right]=\left[\bar{\partial}_{B}, L\right]=\left[\partial_{B}^{*}, \Lambda\right]=\left[\bar{\partial}_{B}^{*}, \Lambda\right] \\
& =\left[L, L_{\eta}\right]=\left[\Lambda, \Lambda_{\eta}\right]=\left[L_{\eta}, \Lambda\right] .
\end{aligned}
$$

One finds $\left\{L_{\eta}, \Lambda_{\eta}\right\}=1$ by direct calculation using the decomposition $\alpha=\alpha_{H}+L_{\eta} \alpha_{V}$. Finally, $\left[d^{0}, \partial_{B}\right]=\partial_{B}+L \Lambda_{\eta}$.

The most involved calculation is that of the commutator

$$
\left[\Lambda, \bar{\partial}_{B}\right]=-\imath \partial_{B}^{*}+\imath L_{\eta} \Lambda+\left(n-d^{0}\right) \Lambda_{\eta}
$$

Before we turn to the proof, let us try to interpret this result as a generalization of the Kähler case $[\Lambda, \bar{\partial}]=-\imath \partial^{*}$. The naive guess $\left[\Lambda, \bar{\partial}_{B}\right] \stackrel{?}{=}-\imath \partial_{B}^{*}$ cannot be correct since the left hand side maps $\left[\Lambda, \bar{\partial}_{B}\right]: \Lambda^{*} D^{*} \rightarrow \Lambda^{*} D^{*}$ while $\partial_{B}^{*}: \Lambda^{*} D^{*} \rightarrow \Lambda^{*} D^{*} \wedge(1 \oplus \eta)$. Similarly, the right hand side annihilates $\eta$ while the left hand side does not. One can guess the correct result by considering the action of both sides on $J$ and $\eta$, adding suitable terms on the right hand side to achieve equality.

The proof of (1.11) is once again an elaboration on the proof for Kähler manifolds in [1]. Let us first consider horizontal forms. Here, it is sufficient to explicitly evaluate the action of (1.11) $L^{i} \alpha$ for $\alpha \in P^{k}$; the result will generalize for generic elements of $\Lambda^{*} D^{*}$ due to Lefschetz decomposition. Furthermore one applies Lefschetz decomposition to $\bar{\partial}_{B} \alpha=\alpha_{0}+L \alpha_{1}+L^{2} \alpha_{2}+\ldots$. We have $\alpha \in P^{k}$ and thus $0=\sum_{j} L^{n-k+1+j} \alpha_{j}$ and finally $L^{n-k+1+j} \alpha_{j}=0$. Using equation (1.9) it follows that most of the $\alpha_{j}$ vanish and $\bar{\partial}_{B} \alpha=\alpha_{0}+L \alpha_{1}$.

Using (1.8) one finds

$$
\left[\Lambda, \bar{\partial}_{B}\right] L^{i} \alpha=-i L^{i-1} \alpha_{0}-(k+i-n-1) L^{i} \alpha_{1}
$$

Similarly, using $\bar{\partial}_{B} \boldsymbol{I}(\alpha)=\imath \boldsymbol{I}\left(\bar{\partial}_{B} \alpha\right)$ and $\boldsymbol{I}^{2}\left(\bigwedge^{k} D^{*}\right)=(-1)^{k}$ as well as (1.6) and (1.10)

$$
\star \bar{\partial}_{B} \star L^{i} \alpha=\imath(-1)^{k^{2}}\left[\Lambda, \bar{\partial}_{B}\right] L^{i} \alpha-(-1)^{k} L_{\eta}\left[L^{i}, \Lambda\right] \alpha .
$$


Finally,

$$
\left.\left[\Lambda, \bar{\partial}_{B}\right]\right|_{\Lambda^{*} D^{*}}=-\imath \partial_{B}^{*}+\imath L_{\eta} \Lambda
$$

To study vertical forms, we consider $L_{\eta} L^{i} \alpha$. Again $\alpha \in P^{k}$ and $\bar{\partial}_{B} \alpha=\alpha_{0}+L \alpha_{1}$. Then

$$
\left[\Lambda, \bar{\partial}_{B}\right] L_{\eta} L^{i} \alpha=i L_{\eta} L^{i-1} \alpha_{0}+(k+i-n-1) L_{\eta} L^{i} \alpha_{1}+[n-(2 i+k)] L^{i} \alpha
$$

Note that $2 i+k$ is the degree of $L^{i} \alpha$. Furthermore,

$$
\star \bar{\partial}_{B} \star L_{\eta} L^{i} \alpha=(-1)^{k^{2}+1} \imath \times\left[i L_{\eta} L^{i-1} \alpha_{0}+(k+i-n-1) L_{\eta} L^{i} \alpha_{1}\right] .
$$

In total,

$$
\left[\Lambda, \bar{\partial}_{B}\right]\left(L_{\eta} L^{i} \alpha\right)=\left\{-\imath \partial_{B}^{*}+[n-(2 i+k)] \Lambda_{\eta}\right\}\left(L_{\eta} L^{i} \alpha\right) .
$$

Since $L_{\eta} \Lambda\left(L_{\eta} L^{i} \alpha\right)=0$, we can add or subtract $\imath L_{\eta} \Lambda$. Therefore it is consistent to combine the results on horizontal and vertical forms into the overall result (1.11). An identical calculation or complex conjugation give $\left[\Lambda, \partial_{B}\right]$. This completes the proof.

We can compute the computator of the adjoints $\left(\alpha \in \Omega_{\mathbb{C}}^{p}\right)$ :

$$
\left[L, \partial_{B}^{*}\right] \alpha=(-1)^{p}\left[-\imath \star \partial_{B}^{*} \star+\imath \star L_{\eta} \Lambda \star+\star\left(n-d^{0}\right) \Lambda_{\eta} \star\right] \alpha_{p} .
$$

With $\star\left(n-d^{0}\right) \star=\left(d^{0}-n\right), \star \partial_{B}^{*} \star \alpha=(-1)^{p+1} \bar{\partial}_{B} \alpha$, and $\star L_{\eta} \Lambda \star \alpha=(-1)^{p+1} \Lambda_{\eta} L \alpha$ one finds

$$
\begin{aligned}
& {\left[L, \partial_{B}^{*}\right]=\imath \bar{\partial}_{B}-\imath \Lambda_{\eta} L+\left(d^{0}-n\right) L_{\eta},} \\
& {\left[L, \bar{\partial}_{B}^{*}\right]=-\imath \partial_{B}+\imath \Lambda_{\eta} L+\left(d^{0}-n\right) L_{\eta} .}
\end{aligned}
$$

The calculation of the anticommutator $\left\{\Lambda_{\eta}, \bar{\partial}_{B}\right\}$ is considerably simpler. Consider again $\alpha \in P^{k}$ with $\bar{\partial}_{B} \alpha=\alpha_{0}+L \alpha_{1}$. Then $\Lambda_{\eta} \bar{\partial}_{B} \alpha=0$ and $\bar{\partial}_{B} \Lambda_{\eta} \alpha=0$. The next step is only slightly more complicated: $\Lambda_{\eta} \bar{\partial}_{B} L_{\eta} \alpha=-\bar{\partial}_{B} \alpha, \bar{\partial}_{B} \Lambda_{\eta} L_{\eta} \alpha=\bar{\partial}_{B} \alpha$ and thus $\left\{\Lambda_{\eta}, \bar{\partial}_{B}\right\}=0$. Similarly $\left\{\Lambda_{\eta}, \partial_{B}\right\}=0$. The adjoint anticommutator vanishes, too.

This concludes the calculation of the identities. The (anti-) commutators allow us to express $\Delta=d^{*} d+d d^{*}$ in terms of $\Delta_{\bar{\partial}_{B}}=\bar{\partial}_{B}^{*} \bar{\partial}_{B}+\bar{\partial}_{B} \bar{\partial}_{B}^{*}$. The decomposition (1.3) yields

$$
\Delta=\Delta_{\partial_{B}}+\Delta_{\bar{\partial}_{B}}+\left\{\partial_{B}, \bar{\partial}_{B}^{*}\right\}+\left\{\bar{\partial}_{B}, \partial_{B}^{*}\right\}-£_{\xi}^{2}
$$

Then, using $\left\{\partial_{B}, \bar{\partial}_{B}^{*}\right\}=\left\{\partial_{B}, L_{\eta} \Lambda\right\}+\imath \partial_{B} \Lambda_{\eta}$ one shows that

$$
\Delta_{\partial_{B}}=\Delta_{\bar{\partial}_{B}}-2 \imath\left(n-d^{0}\right) £_{\xi}+\left\{\partial_{B}-\bar{\partial}_{B}, L_{\eta} \Lambda\right\}-\imath\left(\partial_{B}+\bar{\partial}_{B}\right) \Lambda_{\eta},
$$

which leads to

$$
\Delta=2 \Delta_{\bar{\partial}_{B}}-2 \imath\left(n-d^{0}\right) £_{\xi}-£_{\xi}^{2}+2\left\{\partial_{B}, L_{\eta} \Lambda\right\}-2 \imath \bar{\partial}_{B} \Lambda_{\eta}
$$

Application of $\left\{\partial_{B}, L_{\eta} \Lambda\right\}=\imath L_{\eta} \bar{\partial}_{B}^{*}+\left(n-d^{0}\right) L_{\eta} \Lambda_{\eta}+L \Lambda$ completes the proof of (1). 


\subsection{Beyond Kähler identities?}

Since we found Sasaki-Einstein equivalents of both $\Delta=2 \Delta_{\bar{\partial}}$ and the Kähler identities, it is tempting to ask how much more of Kähler geometry can be generalized. For example, since $\Delta_{\bar{\partial}}$ is self-adjoint and elliptic, one can show that $\Omega_{\mathbb{C}}^{k}=\mathcal{H}^{k} \oplus \Delta_{\bar{\partial}}\left(\Omega_{\mathbb{C}}^{k}\right)$ which implies Hodge's theorem. Similarly, the relation between the de Rham and Hodge Laplacians allows for an isomorphism between the respective spaces of harmonic forms. However, it turns out that $\Delta_{\bar{\partial}_{B}}$ is not elliptic. We will sketch the calculation leading to this result. Recall that $\Delta_{\bar{\partial}_{B}}$ is elliptic if the symbol $\sigma_{\Delta_{\bar{o}_{B}}} \in \operatorname{Hom}\left(\Omega_{\mathbb{C}}^{k}, \Omega_{\mathbb{C}}^{k}\right) \otimes S^{2}\left(T^{*} S\right)$ maps any non-zero $\omega \in T^{*} S$ to an automorphism on $\Omega_{\mathbb{C}}^{k}$. When calculating the symbol one essentially keeps only those terms of $\Delta_{\bar{\partial}_{B}}$ that are of highest order in derivatives. In the context of the tangential CauchyRiemann operator, this means that $\partial_{B}$ and $\bar{\partial}_{B}$ can be taken to be anticommuting and that the overall result is essentially the same as for the symbol of the Dolbeault Laplacian on a Kähler manifold, provided one substitutes $\partial_{z^{i}} \mapsto \partial_{z^{i}}-\eta\left(\partial_{z^{i}}\right) £_{\xi}$. Therefore, $\sigma_{\Delta_{\bar{\partial}_{B}}}(\xi)=0$ and $\Delta_{\bar{\partial}_{B}}$ is not elliptic, yet transversally elliptic [11]. Tievsky's discussion of a transverse Laplacian $\Delta_{T}$ arrives at a similar result. In that case, it turns out that $\Delta_{T}-\left(\Lambda_{\eta} d\right)^{2}$ is elliptic. A similar result should hold here, possibly after replacing $\Lambda_{\eta} d$ with $£_{\xi}$ [9]. El Kacimi-Alaoui has studied elliptic operators acting on basic forms [12].

\section{Motivation and applications}

Both equation (1) as well as the identities in appendix A find application in the AdS/CFT correspondence. Freund-Rubin compactification on Sasaki-Einstein manifolds yields supergravity duals of superconformal field theories [13-17]. The AdS/CFT dictionary links the conformal energy of SCFT operators to the spectrum of $\Delta$, their $R$-charge to that of the Lie-derivative along the Reeb vector, $£_{\xi}$. The conformal energy, $R$-charge, and spin of any SCFT operator have to satisfy the unitarity bounds [18], which should be reflected on the supergravity side in the spectrum of $\Delta$. We will argue shortly that equation (1) allows us to re-derive the unitarity bounds from supergravity when considered in conjunction with the calculations in $[19,20]$.

First, note that the Kähler-like identities allow for a study of the eigenmodes of $\Delta$. In the case where the Sasaki-Einstein manifold has a coset structure, this has been done using harmonic analysis [21]. [19, 20] obtained the structure of the Kaluza-Klein spectrum of generic Sasaki-Einstein manifolds using a construction similar to that in [22], which can be nicely summarized in terms of the identities in appendix A: given any eigen $k$-form $\omega$ of $\Delta$, one diagonalizes the action of $\Delta$ on the $k+1$-forms $\left\{\partial_{B} \omega, \bar{\partial}_{B} \omega, L_{\eta} \omega, L \omega, \partial_{B} \bar{\partial}_{B} \omega, \ldots\right\}$. The resulting eigenstates fill out representations of the superconformal algebra, equivalence classes in Kohn-Rossi cohomology groups $H_{\bar{\partial}_{B}}^{p, q}(S E)$ correspond to short multiplets. Whereas the original calculations were based on a rather tiresome direct approach, the methods developed in this note are expected to simplify that kind of anlysis considerably.

With this in mind, we turn to the spectral problem for $\Delta$. Consider a $k$-form $\omega$ with $£_{\xi} \omega=\imath q, q \geq 0$, and $d^{0} \leq n$. All terms on the right hand side of (1) are positive definite except for the mixed term $M=\imath\left(L_{\eta} \bar{\partial}_{B}^{*}-\bar{\partial}_{B} \Lambda_{\eta}\right)=N+N^{*}$. $M$ is self-adjoint and its 
spectrum is real. Moreover, $N^{2}=0$ and $N\left(\bigwedge^{*} D^{*}\right) \subset \wedge^{*} D^{*} \wedge \eta$ and $N\left(\bigwedge^{*} D^{*} \wedge \eta\right)=0$. That is, $N$ maps horizontal to vertical forms and annihilates the latter. $N^{*}$ behaves accordingly and it follows that $\langle\omega, M \omega\rangle$ vanishes if $\omega$ is horizontal or vertical. This is also the case if $\omega$ is neither horizontal nor vertical yet holomorphic. ${ }^{4}$ As long as we restrict to one of these cases, (1) takes the form of a bound on the spectrum of $\Delta$.

This was conjectured and partially shown in the context of the calculations of the superconformal index $[23,24]$ in $[19,20]$. Here, the spectrum was constructed from primitive elements of $\Omega^{p, q}$. For such forms, (1) implies

$$
\Delta \geq q^{2}+2 q\left(n-d^{0}\right)
$$

with equality if and only if $\bar{\partial}_{B} \omega=\bar{\partial}_{B}^{*} \omega=0$. In the Kähler case, the latter of these is implied by transversality $-d^{*} \omega=0$. Here however, $d^{*} \omega=0$ leads only to the vanishing of the horizontal component of $\bar{\partial}_{B}^{*} \omega$. Indeed,

$$
\partial_{B}^{*} \omega=\imath L_{\eta} \Lambda \omega, \quad \bar{\partial}_{B}^{*} \omega=-\imath L_{\eta} \Lambda \omega,
$$

which vanishes since $\omega$ was assumed to be primitive. Assuming that every element of $H_{\bar{\partial}_{B}}^{p, q}(S)$ has a representative closed under $\bar{\partial}_{B}^{*}$, the bound (2.1) is saturated on the elements of $H_{\bar{\partial}_{B}}^{p, q}(S)$. These are the forms that correspond to the short multiplets in the SCFT, and (2.1) together with the expressions for the derived eigenmodes of $\Delta$ given in $[19,20]$ allows to recover the unitarity bounds from supergravity. Note that (2.1) and a precursor to (1) were already conjectured in those references. Furthermore, the appendix of [20] contains an argument that every element of $H_{\bar{\partial}_{B}}^{p, q}(S)$ is either primitive, carrying zero charge, or both. For the cases of interest in the context of that paper it turned out that all elements are primitive.

A further application of (1) is the stability analysis of Pilch-Warner solutions in [25]. In the absence of general theorems concerning Laplace operators on Sasaki-Einstein manifolds, the authors constructed explicitly examples of primitive, basic $(1,1)$-forms whose existence renders these solutions perturbatively unstable. Assuming that the calculations in [25] generalize to generic transverse forms, our results might allow for a continuation of their analysis to manifolds where explicit constructions are not feasible.

Finally, it would be interesting to extend the results presented here beyond the SasakiEinstein case. As long as there is a dual SCFT, there is a unitarity bound meaning that there should be some equivalent of (1) or at least (2.1).

\section{Acknowledgments}

I would like to thank Richard Eager and Yuji Tachikawa for the collaborations and discussions that lead to the results presented here. I would also like to thank Jyotirmoy Bhattacharya for discussions and James Sparks for correspondence related to this project. Finally, I would like to thank the organizers of Strings 2013 and the joint YITP/KIAS workshop where most of this paper was written.

\footnotetext{
${ }^{4}$ In the remainder of this discussion, the term holomorphic is meant in respect to the tangential CauchyRiemann operator $\bar{\partial}_{B}$.
} 


\begin{tabular}{|c|ccccccc|}
\hline & $L_{\eta}$ & $\Lambda_{\eta}$ & $L$ & $\Lambda$ & $\partial_{B}^{*}$ & $\bar{\partial}_{B}^{*}$ & $\bar{\partial}_{B}$ \\
\hline$\partial_{B}$ & $\left\{\partial_{B}, L_{\eta}\right\}=L$ & $\left\{\partial_{B}, \Lambda_{\eta}\right\}=0$ & {$\left[\partial_{B}, L\right]=0$} & {$\left[\partial_{B}, \Lambda\right]$} & $\Delta_{\partial_{B}}$ & $\left\{\partial_{B}, \bar{\partial}_{B}^{*}\right\}$ & $\left\{\partial_{B}, \bar{\partial}_{B}\right\}=-2 L £_{\xi}$ \\
$\bar{\partial}_{B}$ & $\left\{\bar{\partial}_{B}, L_{\eta}\right\}=L$ & $\left\{\bar{\partial}_{B}, \Lambda_{\eta}\right\}=0$ & {$\left[\bar{\partial}_{B}, L\right]=0$} & {$\left[\bar{\partial}_{B}, \Lambda\right]$} & $\left\{\bar{\partial}_{B}, \partial_{B}^{*}\right\}$ & $\Delta_{\bar{\partial}_{B}}$ & \\
$\bar{\partial}_{B}^{*}$ & $\left\{\bar{\partial}_{B}^{*}, L_{\eta}\right\}=0$ & $\left\{\bar{\partial}_{B}^{*}, \Lambda_{\eta}\right\}=\Lambda$ & {$\left[\bar{\partial}_{B}^{*}, L\right]$} & {$\left[\bar{\partial}_{B}^{*}, \Lambda\right]=0$} & $\left\{\bar{\partial}_{B}^{*}, \partial_{B}^{*}\right\}=2 \Lambda £_{\xi}$ & & \\
$\partial_{B}^{*}$ & $\left\{\partial_{B}^{*}, L_{\eta}\right\}=0$ & $\left\{\partial_{B}^{*}, \Lambda_{\eta}\right\}=\Lambda$ & {$\left[\partial_{B}^{*}, L\right]$} & {$\left[\partial_{B}^{*}, \Lambda\right]=0$} & & \\
$\Lambda$ & {$\left[\Lambda, L_{\eta}\right]=0$} & {$\left[\Lambda, \Lambda_{\eta}\right]=0$} & {$[\Lambda, L]=\left(n-d^{0}\right)$} & & & & \\
$L$ & {$\left[L, L_{\eta}\right]=0$} & {$\left[L, \Lambda_{\eta}\right]=0$} & & & & \\
$\Lambda_{\eta}$ & $\left\{\Lambda_{\eta}, L_{\eta}\right\}=1$ & & & & & \\
\hline
\end{tabular}

Table 1. The Kähler-like identities.

\section{A The identities}

Table 1 summarizes the various (anti-) commutators. The more involved ones that do not fit in the table are listed in equation (A.1).

$$
\begin{aligned}
{\left[\partial_{B}, \Lambda\right] } & =-\imath \bar{\partial}_{B}^{*}+\imath L_{\eta} \Lambda-\left(n-d^{0}\right) \Lambda_{\eta} \\
{\left[\bar{\partial}_{B}, \Lambda\right] } & =\imath \partial_{B}^{*}-\imath L_{\eta} \Lambda-\left(n-d^{0}\right) \Lambda_{\eta}, \\
{\left[\partial_{B}^{*}, L\right] } & =-\imath \bar{\partial}_{B}+\imath \Lambda_{\eta} L-\left(d^{0}-n\right) L_{\eta}, \\
{\left[\bar{\partial}_{B}^{*}, L\right] } & =\imath \partial_{B}-\imath \Lambda_{\eta} L-\left(d^{0}-n\right) L_{\eta}, \\
\left\{\partial_{B}, \bar{\partial}_{B}^{*}\right\} & =\imath\left(L_{\eta} \bar{\partial}_{B}^{*}+\partial_{B} \Lambda_{\eta}\right)+\left(n-d^{0}\right) L_{\eta} \Lambda_{\eta}+L \Lambda, \\
\left\{\bar{\partial}_{B}, \partial_{B}^{*}\right\} & =-\imath\left(L_{\eta} \partial_{B}^{*}+\bar{\partial}_{B} \Lambda_{\eta}\right)+\left(n-d^{0}\right) L_{\eta} \Lambda_{\eta}+L \Lambda .
\end{aligned}
$$

Open Access. This article is distributed under the terms of the Creative Commons Attribution License (CC-BY 4.0), which permits any use, distribution and reproduction in any medium, provided the original author(s) and source are credited.

\section{References}

[1] D. Huybrechts, Complex geometry: an introduction, Springer, Germany (2005).

[2] C. Voisin, Hodge theory and complex algebraic geometry, volume 1, Cambridge University Press, Cambridge U.K. (2008).

[3] H. Lewy, On the local character of the solutions of an atypical linear differential equation in three variables and a related theorem for regular functions of two complex variables, Ann. Math. 64 (1956) 514.

[4] J. Kohn and H. Rossi, On the extension of holomorphic functions from the boundary of a complex manifold, Ann. Math. 81 (1965) 451.

[5] G. Folland and J. Kohn, The Neumann problem for the Cauchy-Riemann complex, Princeton University Press, Princeton U.S.A. (1972).

[6] S. Yau, Kohn-Rossi cohomology and its application to the complex plateau problem. I, Ann. Math. 113 (1981) 67.

[7] H. Luk and S. Yau, Kohn-Rossi cohomology and its application to the complex plateau problem. II, J. Diff. Geom. 77 (2007) 135. 
[8] R. Du and S. Yau, Kohn-Rossi cohomology and its application to the complex plateau problem. III, J. Diff. Geom. 90 (2012) 251.

[9] A.M. Tievsky, Analogues of Kähler geometry on Sasakian manifolds, Ph.D. thesis, MIT, U.S.A. (2008).

[10] J. Sparks, Sasaki-Einstein manifolds, Surveys Diff. Geom. 16 (2011) 265 [arXiv:1004.2461] [INSPIRE].

[11] M. Atiyah, Elliptic operators and compact groups, Lecture Notes in Mathematics volume 401, Springer, Berlin, Germany (1974).

[12] A. El Kacimi-Alaoui, Opérateurs transversalement elliptiques sur un feuilletage riemannien et applications, Comp. Math. 73 (1990) 57.

[13] A. Kehagias, New type IIB vacua and their F-theory interpretation, Phys. Lett. B 435 (1998) 337 [hep-th/9805131] [INSPIRE].

[14] I.R. Klebanov and E. Witten, Superconformal field theory on three-branes at a Calabi-Yau singularity, Nucl. Phys. B 536 (1998) 199 [hep-th/9807080] [INSPIRE].

[15] B.S. Acharya, J. Figueroa-O'Farrill, C. Hull and B.J. Spence, Branes at conical singularities and holography, Adv. Theor. Math. Phys. 2 (1999) 1249 [hep-th/9808014] [INSPIRE].

[16] D.R. Morrison and M.R. Plesser, Nonspherical horizons. 1, Adv. Theor. Math. Phys. 3 (1999) 1 [hep-th/9810201] [INSPIRE].

[17] D. Martelli and J. Sparks, Toric geometry, Sasaki-Einstein manifolds and a new infinite class of AdS/CFT duals, Commun. Math. Phys. 262 (2006) 51 [hep-th/0411238] [InSPIRE].

[18] S. Minwalla, Restrictions imposed by superconformal invariance on quantum field theories, Adv. Theor. Math. Phys. 2 (1998) 781 [hep-th/9712074] [INSPIRE].

[19] R. Eager, J. Schmude and Y. Tachikawa, Superconformal indices, Sasaki-Einstein manifolds and cyclic homologies, arXiv:1207.0573 [INSPIRE].

[20] R. Eager and J. Schmude, Superconformal indices and M2-branes, arXiv:1305.3547 [INSPIRE].

[21] A. Ceresole, G. Dall'Agata and R. D'Auria, KK spectroscopy of type IIB supergravity on $A d S_{5} \times T^{11}, J H E P 11$ (1999) 009 [hep-th/9907216] [INSPIRE].

[22] C. Pope, Kähler manifolds and quantum gravity, J. Phys. A 15 (1982) 2455.

[23] J. Kinney, J.M. Maldacena, S. Minwalla and S. Raju, An index for 4 dimensional super conformal theories, Commun. Math. Phys. 275 (2007) 209 [hep-th/0510251] [INSPIRE].

[24] J. Bhattacharya, S. Bhattacharyya, S. Minwalla and S. Raju, Indices for superconformal field theories in 3, 5 and 6 dimensions, JHEP 02 (2008) 064 [arXiv:0801.1435] [INSPIRE].

[25] K. Pilch and I. Yoo, On perturbative instability of Pope-Warner solutions on Sasaki-Einstein manifolds, JHEP 09 (2013) 124 [arXiv: 1305.0295] [INSPIRE]. 